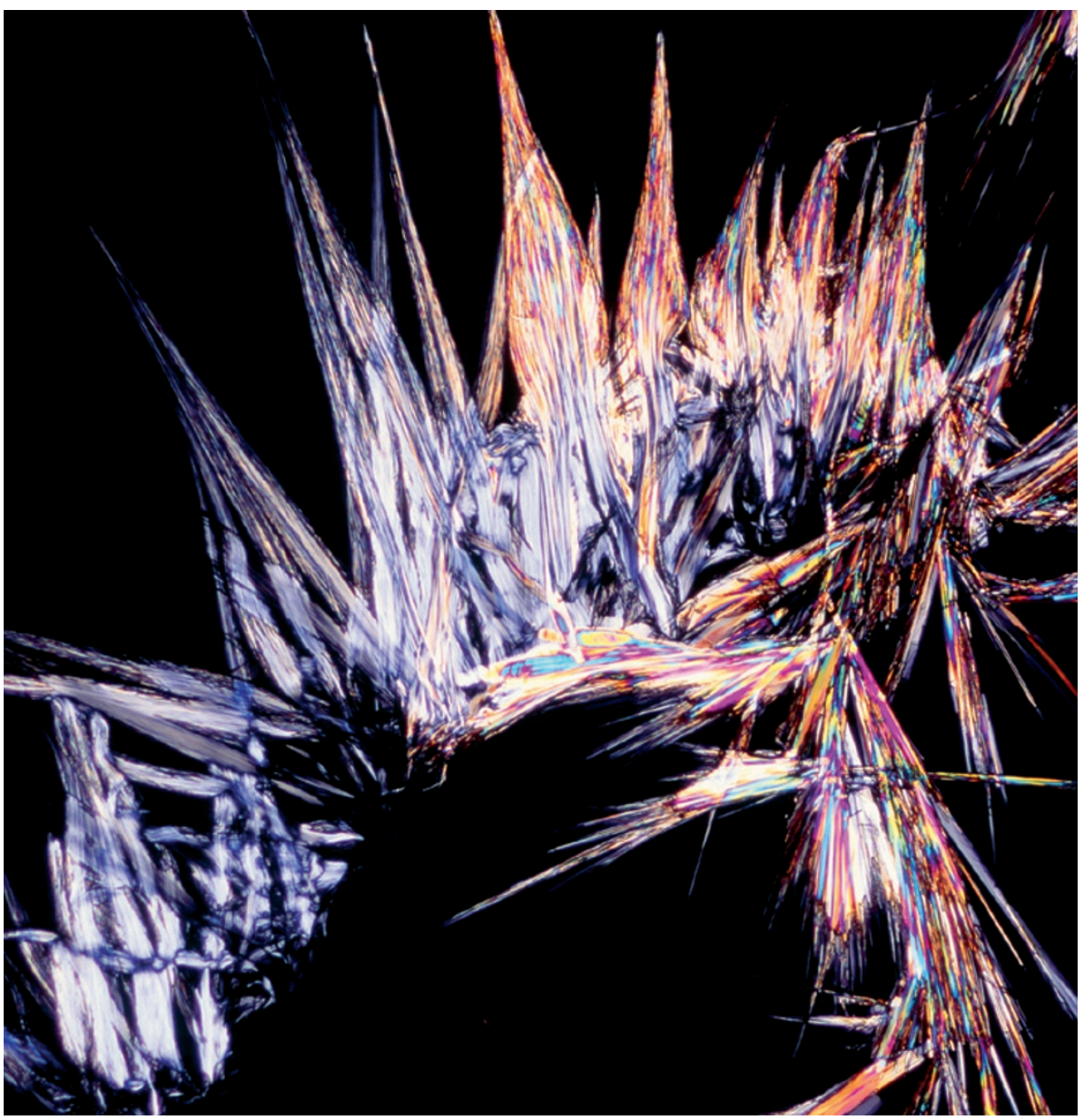

Cholesterol crystals accumulate inside plaques - a build-up causes cardiovascular disease.

PATHOLOGY

\title{
At the heart of the problem
}

\section{Research is illuminating the molecular mechanisms that can cascade into debilitating heart disease.}

\section{BY CASSANDRA WILLYARD}

A fter the end of the Second World War, the number of deaths related to heart disease in the United States skyrocketed. At that time, the causes of heart disease weren't well understood. So in 1948, the US Public Health Service launched a landmark study in Framingham, Massachusetts, and recruited more than 5,000 of its townspeople. The Framingham Heart Study helped identify many of the major risk factors: smoking, high cholesterol, high blood pressure, diabetes, obesity and lack of exercise. They also identified factors that reduce the risk of heart disease.
In recent decades, researchers have begun untangling the molecular pathways that underlie these risk factors. Even seemingly straightforward relationships, such as the link between high cholesterol and heart disease, are turning out to be more complex than previously believed, which has researchers questioning even the most basic assumptions.

Heart disease is an umbrella term that includes a wide variety of ailments. By far the most common, however, is coronary heart disease, which occurs when fatty plaque builds up in the arteries that feed the heart a process called atherosclerosis. Plaque can restrict blood flow, but the bigger problem occurs when a plaque ruptures, spilling its contents into the bloodstream and causing a clot to form. A clot that cuts off blood flow to the heart will cause a heart attack. If the clot blocks flow to the brain, the result is a stroke.

Cholesterol is a major component of plaque. "The body itself cannot break down cholesterol. It has to store it or export it, or turn it into something else, like a hormone," says Frank Sacks, a specialist in cardiovascular disease prevention at Harvard School of Public Health in Boston, Massachusetts. "So if cholesterol goes into an artery, it's stuck there." Lipoproteins (conglomerations of lipids and proteins) shuttle cholesterol through the body. A surplus of low-density lipoprotein (LDL), often called 'bad cholesterol', can lodge in artery walls, promoting the formation of plaque and increasing the risk of a heart attack or stroke.

\section{THE COMPLEX ROLE OF HDL}

High-density lipoprotein (HDL), a larger molecule, clears away cholesterol-laden plaque from the artery walls and carries it to the liver for disposal. Epidemiological studies consistently show that people who have high levels of HDL cholesterol in their blood have fewer heart attacks. More recent studies, however, suggest that HDL might not be universally protective. "You lower LDL, you reduce heart disease any way you do it," Sacks says. But raising HDL is not always beneficial. "We know there's something protective about HDL," Sacks says. "We cannot really identify what it is."

Part of the problem is that HDL is a mix of molecules that differ in size and composition. Sacks and his colleagues speculated that some types of HDL might be more protective than others. Their previous work pointed them to a small protein found on the surface of some lipoproteins called apolipoprotein (apo) C-III (ref. 1). Studies show that LDL bearing apoCIII promotes plaque build-up in the arteries. Sacks wondered what effect the protein might have on the function of HDL.

So Sacks and his colleagues examined blood samples collected as part of two large epidemiological studies: the Nurses' Health Study, which included about 122,000 female nurses, and the Health Professionals Follow-up Study, which included about 52,000 men. Not surprisingly, Sacks found that HDL was protective; individuals who had higher levels had fewer heart attacks ${ }^{2}$. However, when he focused on HDL with apoC-III, which made up about 10-15\% of total HDL, he uncovered an adverse effect in both study groups. When they pooled the two studies, evidence for the adverse effect grew even stronger. HDL with apoC-III "actually predicted a higher, not a lower, rate of heart disease", Sacks says. When the team excluded this harmful HDL, the protective effect of the remaining HDL grew even stronger.

If HDL were directly involved in protecting against heart disease, then individuals who 
have genetic variants associated with increased HDL production should have fewer heart attacks. Indeed, variants that lower LDL are consistently associated with a reduced risk of heart attacks. But the reverse doesn't seem to hold true for HDL. A group of researchers examined the effect of a genetic variant that boosts HDL cholesterol levels, but individuals with this variant did not have fewer heart attacks ${ }^{3}$.

These and other studies suggests that "we still need to learn more about HDL biology and recognize that it's a complex molecule in order to be sure that we develop the best therapeutic strategy," says Gary Gibbons, director of the US National Heart, Lung, and Blood Institute in Bethesda, Maryland.

\section{AN INFLAMED HEART}

The mechanisms that lead to atherosclerosis are just as complex as the mechanisms that protect against it. For example, research over the past three decades suggests that inflammation plays an intermediary role.

In the 1980s, scientists found that plaque contains macrophages - immune cells that play a role in inflammation. Researchers now suspect that the presence of macrophages results from a cascade of events. The LDL in artery walls prompts endothelial cells, which line the vessels, to produce sticky molecules that snag macrophage precursor cells, monocytes, from the blood. At the same time, endothelial and smooth muscle cells begin to pump out chemicals that attract monocytes. These newly recruited monocytes enter the arterial wall and mature into macrophages, which gorge on LDL cholesterol and balloon in size. "You can't have atherosclerotic plaque without having cholesterol-laden macrophages in your artery wall. That's the major cell type that accumulates," explains Stanley Hazen, an endocrinologist who is head of preventive cardiology and cardiac rehabilitation at the Cleveland Clinic in Ohio. Because their interior is dotted with fatty globs of cholesterol, pathologists call them foam cells. These foam cells are the hallmark of atherosclerotic plaque; they and other immune cells produce chemical signals that prompt smooth muscle cells to migrate to the top of the plaque and form a tough cap. As foam cells accumulate, some die. The cap keeps this pool of living and dead foam cells safely walled off from the blood stream.

Inflammation seems to increase the likelihood of plaque rupture, which sparks the formation of potentially lethal clots. Collagen and other materials make the cap strong. But inflammatory mediators in the plaque can inhibit collagen synthesis and ramp up the production of enzymes that break down collagen, leading to a thin and weakened fibrous cap, says Peter Libby, chief of cardiovascular medicine at Brigham and Women's Hospital in Boston. Libby's lab and other research groups have also shown that inflammatory mediators can prompt macrophages in the plaque to

\section{PROBLEMS FROM PLAQUE}

Various cells and cholesterol can interact to block arteries, which is a major cause of heart disease.

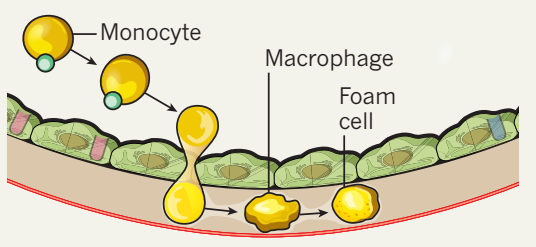

Blockage begins when the arterial surface captures immune cells (monocytes), which enter the wall and become macrophages that turn into foam cells.

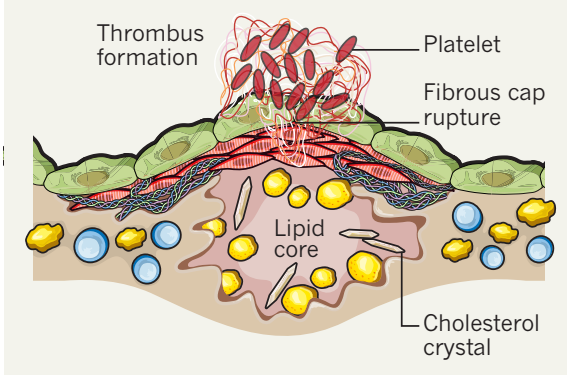

The foam cells and cholesterol crystals collect in a lipid core.

2 A rupture in the arterial wall releases the material in the plaque, which reduces blood flow in the artery.

churn out tissue factor, a protein that promotes clotting. "So not only is inflammation involved in the very first steps of atherosclerosis, but also in the ultimate complications," Libby says.

Libby recently added another subplot to this story ${ }^{4}$. Using mice genetically predisposed to develop plaque in their arteries, he and his colleagues showed that a heart attack worsens atherosclerosis by boosting the release of stem cells from the bone marrow. These cells travel to the spleen and become monocytes. The researchers propose that the anxiety and pain associated with a heart attack triggers the sympathetic nervous system to boost stem-cell release and that interrupting this chain might help to prevent future heart attacks. The team also found high numbers of stem cells in the spleens of patients who had died of a heart attack, which suggests the process might work in a similar way in humans ${ }^{5}$.

\section{MICROBIAL INFLUENCES}

Some of the factors involved in the development of atherosclerosis are entirely new and unexpected. Hazen stumbled across one of these surprising mechanistic pathways a few years ago while looking for metabolites linked to atherosclerosis. Hazen identified a group of compounds "strikingly associated with cardiovascular risk", he says. Some were metabolites that could only be produced by bacteria. "Once we saw that bacteria were likely involved in the pathway, we started looking to the gut," Hazen says. His focus turned to one compound: trimethylamine $\mathrm{N}$-oxide (TMAO).

Gut microbes do not produce TMAO directly. They convert phosphatidylcholine (a common component of animal products such as meat and eggs) into a foul-smelling gas called trimethylamine; the liver then converts this gas into TMAO. When Hazen's team gave the mice TMAO, "that alone was sufficient to accelerate atherosclerosis", Hazen says. Even the trimethylamine precursor promoted atherosclerosis when the TMAO-producing microbes were present. But the researchers found they could protect against this by killing the mice's gut bacteria with antibiotics.

There are various reasons why TMAO might promote atherosclerosis. Hazen and colleagues found that the metabolite increases the number of receptors on the surface of macrophages that bind to LDL, which makes the cells more prone to gobble up cholesterol ${ }^{6}$. "This pathway sits right at the junction between cholesterol metabolism and inflammation," Hazen says. "It's influencing both in the artery wall."

If microbes are part of the problem, could antibiotics be part of the solution? Hazen points out that a number of randomized controlled trials have tested whether antibiotics can prevent heart disease, but none proved fruitful. The problem might have been that the drugs tested failed to wipe out the TMAO-producing organisms. Microbes can quickly develop resistance. "We do all our mouse studies now not with a single antibiotic, but with a big gorilla cocktail of five different antibiotics," Hazen says. But he doesn't advocate antibiotic cocktails for the prevention of heart disease. Instead he envisages using probiotics - beneficial microorganisms - to promote healthy microflora, or drugs to interrupt the pathway without killing the bacteria.

Much has changed since the launch of the Framingham Heart Study back in the 1940s. Scientists have a far better understanding of the causes of heart disease, and death rates have plummeted. "There has been nothing short of a cardiac revolution," says Michael Lauer, director of the division of cardiovascular sciences at the National Heart, Lung, and Blood Institute. "It's one of the great triumphs of modern medical science." But the precise manner in which our hearts can betray us has not yet been fully revealed.

\section{Cassandra Willyard is a freelance science} writer based in Brooklyn, New York.

1. Sacks, F. M. et al. Circulation 102, 1886-1892 (2000).

2. Jensen, M. K. et al. J. Am. Heart Assoc.1, jah3-e000232 (2012).

3. Voight, B. F. et al. Lancet 380, 572-580 (2012).

4. Dutta, P. et al. Nature 487, 325-329 (2012).

5. Wang, Z. et al. Nature 472, 57-63 (2011)

6. Petersen, T. H. et al. Science 329, 538-541 (2010). 